\title{
Necrotizing Sarcoid Granulomatosis: Possibly Veiled Disease in Endemic Area of Mycobacterial Infection
}

\author{
Yosep Chong · Eun Jung Lee · Chang Suk Kang · Tae-Jung Kim · Jung Sup Song ${ }^{1} \cdot$ Hyosup Shim $^{2}$ \\ Departments of Hospital Pathology and ${ }^{1}$ Internal Medicine, College of Medicine, The Catholic University of Korea, Seoul; \\ 2Department of Pathology, Yonsei University College of Medicine, Seoul, Korea
}

Necrotizing sarcoid granulomatosis (NSG) is a rare granulomatous disease that primarily affects the lung and presents as nodular masses of confluent sarcoid-like granulomas with extensive necrosis and vasculitis. ${ }^{1}$ Proper diagnosis and treatment are challenging for clinicians, radiologists, and pathologists because of the rarity and diagnostic difficulty of this disease..$^{2-4}$ Since it was first described by Liebow, ${ }^{1}$ only 135 cases have been reported. ${ }^{2,4,5}$

In addition to its rarity, the similarity of the clinical, radiological, and pathological features of NSG to other granulomatous diseases, such as granulomatous infection, nodular sarcoidosis, and Wegener's granulomatosis (WG), is the largest obstacle in its proper diagnosis. The initial symptoms are non-specific or frequently do not present at all. ${ }^{2-4}$ Radiologically, NSG can present as cavitary lesions, ill-defined pneumonic consolidations, or even as a solitary nodule or a mass. ${ }^{6,7}$ Pathologically, NSG shares features of sarcoidosis and WG. ${ }^{1,4}$

Thus, it is not surprising that NSG is often easily mistaken for granulomatous infections, such as tuberculosis, especially in endemic areas. Although the general level of hygiene in Korea has dramatically improved, the reported incidence of pulmonary tuberculosis remains the highest among the Organization for Economic Cooperation and Development member countries. ${ }^{89}$ Identification of causal microorganisms is essential for proper treatment of tuberculosis; however, empirical treatment with

Corresponding Author Tae-Jung Kim, MD, PhD

Department of Hospital Pathology, Yeouido St. Mary's Hospital, 10 63-ro Yeongdeungpo-gu, Seoul 150-713, Korea

Tel: +82-2-3779-2157, Fax: +82-2-783-6648, E-mail: kimecho@catholic.ac.kr

Received: February 2, 2015 Revised: April 2, 2015

Accepted: April 16, 2015 anti-tuberculosis medication is often performed in endemic countries like Korea, in spite of tests failing to show any causative microorganisms. ${ }^{9}$

Here, we report the first two cases of NSG in Korean patients, one of whom showed a dramatic response to immediate application of systemic corticosteroids, and the other of whom showed no response to empirical treatment with anti-tuberculosis medication.

\section{CASE REPORT}

The publication of the case information and materials was approved by the institutional review board of The Catholic University of Korea, College of Medicine (SC11ZISE0221).

\section{Case 1}

A 70-year-old man with a history of smoking presented with a three-week history of cough, fever, and chill. He reported no change in body weight, no cyanosis, and no clubbing. Vital signs were stable, and inflammatory markers were slightly increased (erythrocyte sedimentation rate, $90 \mathrm{~mm} / \mathrm{hr}$; C-reactive protein [CRP], $3.52 \mathrm{mg} / \mathrm{L})$.

Radiological examination revealed multifocal ill-defined, nodular consolidations in both lungs, with moderate enlargement of paratracheal and hilar lymph nodes, suggesting granulomatous infection (Fig. 1A, B).

On microscopic examination of the wedge-resected tissue, confluent small granulomas with necrosis of variable sizes were seen in the lung parenchyma. The granulomas were accompanied by central necrosis that was suppurative rather than caseous, palisaded by Langerhans-type giant cells and mononuclear 
lymphohistiocytes. At first glance, granulomatous vasculitis did not seem evident, but on closer observation, mild transmural vasculitis with fibrosis distant from the necrotic area was clearly noted in a significant portion of the lesion (Fig. 2A-D). The differential diagnosis based on pathologic findings included granulomatous diseases of various causes. Special stains for fungus and acid-fast bacilli on the resected tissue and sputum were negative. Tuberculosis-polymerase chain reaction and culture on bronchial washing fluid and peripheral blood were both negative. Serum cryptococcal antigen and $\operatorname{IgG}$ for parasites were all negative.

An extensive battery of autoimmune markers was all negative (antineutrophil cytoplasmic antibody, antinuclear, anti-ds-DNA, anti-sm, anti-SS-A/Ro, anti-Sc172, anti-Jo1, etc.). Serum angiotensin-converting enzyme was unremarkable. With these results, a diagnosis of NSG was highly suspected and treated with oral prednisolone.

After one month of therapy, the patient showed a dramatic improvement of symptoms and chest radiography (Fig. 1C). He has had no symptoms through two years of follow-up.

\section{Case 2}

A 41-year-old non-smoking woman presented with a twoweek history of cough and sputum. Laboratory findings showed only a mild increase in acute inflammatory marker (CRP, 37.4 $\mathrm{mg} / \mathrm{L})$.

Radiologically, multiple nodules with ill-defined margins scattered mainly in the left lower lobe were found with minimal pleural effusion, suggesting hematogenous spread of metastatic cancer, pulmonary lymphoma, or septic pneumonia (Fig. 1D). Hilar lymph nodes were unremarkable.

Histological examination of resected tissue revealed variably-
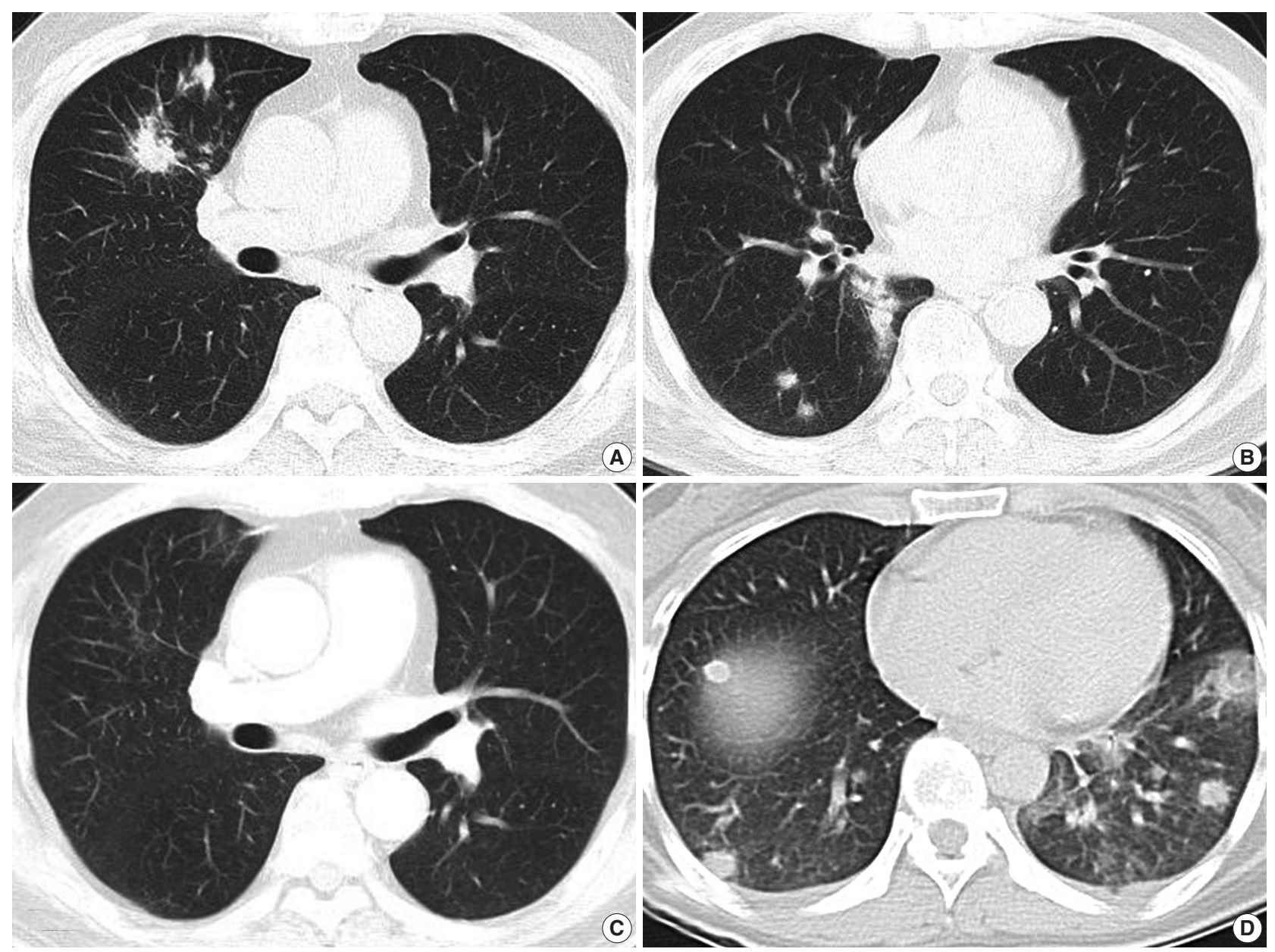

Fig. 1. Radiologic findings. (A, B) Computed tomography (CT) of case 1 shows multiple variably-sized nodular lesions with hilar node enlargement. (C) Follow-up CT after one month of corticosteroid therapy reveals dramatic resolution. (D) CT of case 2 also reveals multiple illdefined nodules. 

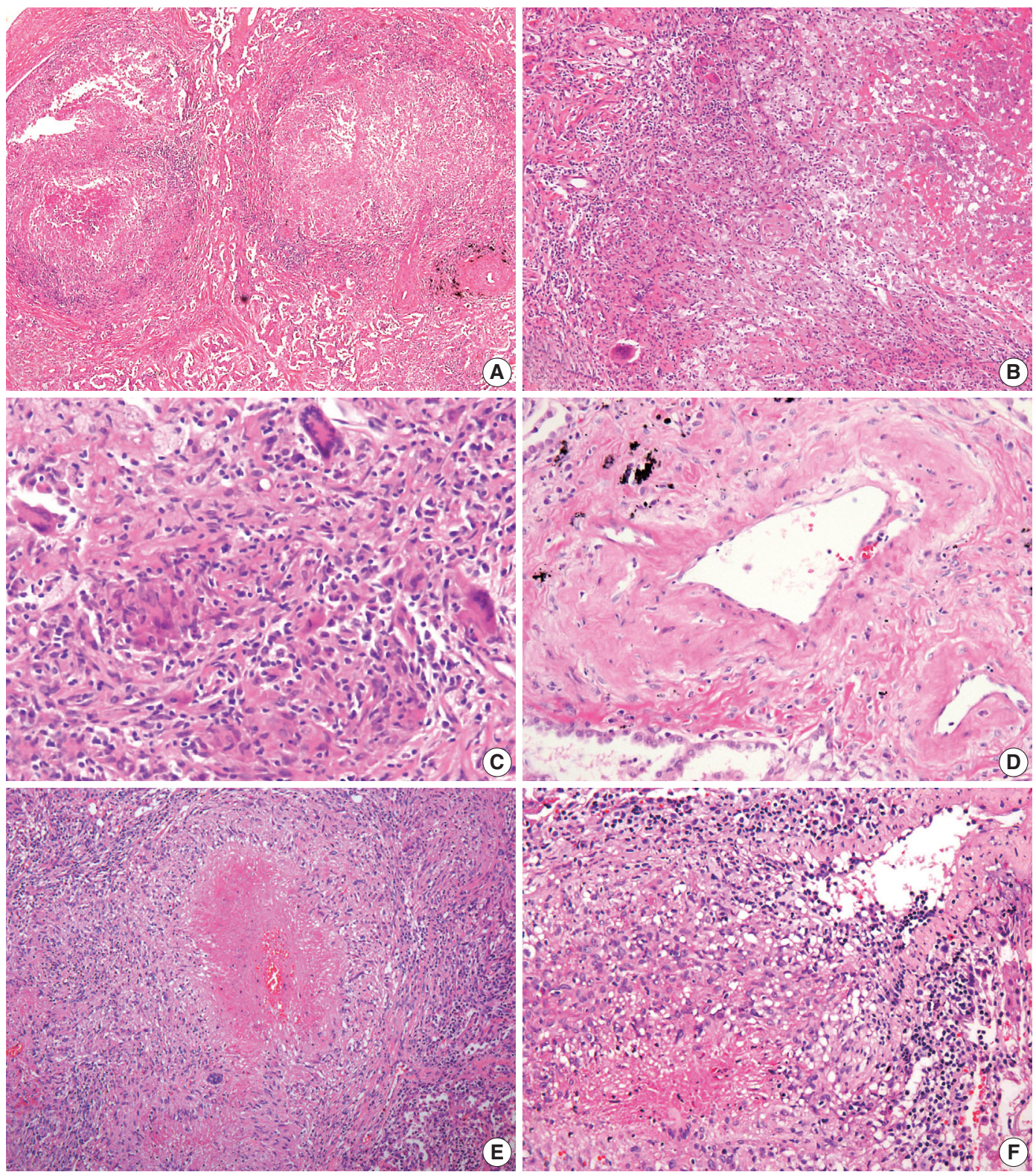

Fig. 2. Microscopic findings. (A-C) Multifocal nodular granulomatous inflammation with extensive central necrosis is replacing normal architecture (case 1). (D) Mild transmural vasculitis distant from necrotic area, i.e., cicatricial-type vasculitis, is observed. (E, F) Multiple confluent granulomas with central caseous necrosis and granulomatous vasculitis are found (case 2).

sized granulomas with varying amounts of suppurative necrosis and frequent granulomatous vasculitis, raising suspicion for sarcoidosis or other kinds of granulomatous vasculitis (Fig. 2E, F). However, autoimmune markers were all negative, and no causative microorganisms were found on various tests.

An anti-tuberculosis treatment regimen was started based on the clinicoradiographic findings in order to exclude the possibility of potential pulmonary tuberculosis. During the first three weeks of anti-tuberculosis treatment, the patient reported no improvement in symptoms. She was treated for another month and slowly improved, irrespective of treatment, and even after its cessation. Bacterial cultures of sputum, blood, and bronchial washing remained negative.

\section{DISCUSSION}

In 1973, Liebow ${ }^{1}$ described three chief features of NSG that differentiate it from sarcoidosis, WG, and other granulomatous infections. First, histologically, NSG appears as sarcoid-like granuloma with vasculitis and necrosis. Second, radiological 
findings include multiple lung nodules without hilar lymphadenopathy. Third, it has a very benign clinical course. However, subsequent reports have since shown that NSG can present with more variable radiologic findings and is commonly accompanied by lymphadenopathy and pleural effusion. ${ }^{3,4}$

Because the symptoms of NSG are often non-specific and the radiologic findings vary widely, the pathological findings are important for accurate diagnosis. If one finds a mixture of pathologic features of pulmonary sarcoidosis and mild to moderate granulomatous vasculitis, combined with negative tests for causative microorganisms, the possibility of NSG can be raised. However, conclusive diagnosis cannot be made on histologic features alone, and the final pathologic diagnosis of NSG should be made after thorough exclusion of the other possible diseases with similar features. Nodular sarcoidosis is excluded by the presence of necrotizing vasculitis and diffuse parenchymal necrosis. ${ }^{4} \mathrm{WG}$ is excluded by the presence of sarcoid-like granulomas and granulomatous vasculitis distant from necrotic areas. ${ }^{4}$ Most importantly, granulomatous infections should be carefully excluded because they can also produce variable vasculitis, extensive necrosis, and sarcoid-like granulomas. ${ }^{3,4}$ Such distinction can be very challenging because most microbiological studies, including those that use the latest technologies such as polymerase chain reaction, can produce false-negative results.

In describing the characteristic pathologic findings of NSG, Katzenstein ${ }^{4}$ has mentioned that transmural vasculitis with fibrosis distant from necrotic area, as seen in the first case of this report, is an important defining finding of NSG. It is important to remember that these findings can be easily overlooked and considered as usual nodular sarcoidosis if pathologists do not pay enough attention to the lung biopsy samples of similar conditions. For this reason, Rosen ${ }^{5}$ suggests in his recent review that NSG is a specific form of sarcoidosis and should be referred to with the diagnostic term sarcoidosis with necrotizing sarcoid granulomatosis pattern. However, since this specific form of sarcoidosis mimics granulomatous infections such as tuberculosis and results in inadequate anti-tuberculosis treatment, the distinction from usual pulmonary sarcoidosis should continue to be emphasized.

According to the guidelines on treatment of tuberculosis by the Centers for Disease Control and Prevention, a four-drug antituberculosis regimen is recommended for the first two months of treatment for patients who are highly suspected as having an active tuberculosis infection based on clinicoradiographical findings, even without isolation of mycobacterium. ${ }^{10}$ The Korean Guidelines for Tuberculosis also recommend empirical chemo- prevention in sputum-negative patients while waiting for the results of culture studies. ${ }^{9}$ Thus, it is inevitable that patients with NSG in Korea will undergo the risks and inconvenience of anti-tuberculosis treatment considering the socioeconomic burden of tuberculosis. However, it should be noted that there may be more unrecognized patients with NSG in Korea, and that they might be overlooked in the endemic setting of tuberculosis, considering that NSG has been reported quite commonly in Japan, the most similar Asian country to Korea. ${ }^{11}$

Collectively, for the accurate recognition of NSG, the following diagnostic steps should be performed. If pathologically suspicious features for NSG are found in the absence of identification of any causative microorganisms and the radiologic findings demonstrate multiple nodules with hilar lymphadenopathy, the possibility of NSG should be considered. If NSG is clinically suspected, the initial two to three weeks of anti-tuberculosis treatment can be used to monitor the disease response. If there is no change in symptoms or radiological findings, then systemic steroid treatment can be applied.

To our knowledge, this is the first case report of NSG in Korean patients, one of whom showed a dramatic response to corticosteroid therapy. A case of NSG was reported in 1997 by Kim et al., ${ }^{12}$ but the evidence for NSG in their report is insufficient because polymerase chain reaction was not used to exclude tuberculosis, the patient was not appropriately treated, and there was no radiographic improvement.

In conclusion, the diagnosis of NSG should be made very carefully in suspected cases based on consistent radiologic and histologic findings and thorough exclusion of possible causative microorganisms.

\section{Conflicts of Interest}

No potential conflict of interest relevant to this article was reported.

\section{REFERENCES}

1. Liebow AA. The J. Burns Amberson lecture: pulmonary angiitis and granulomatosis. Am Rev Respir Dis 1973; 108: 1-18.

2. Corrin B, Nicholson AG. Pathology of the lungs. 3rd ed. New York: Churchill Livingstone/Elsevier, 2011.

3. Leslie KO, Wick MR. Practical pulmonary pathology: a diagnostic approach. 2nd ed. Philadelphia: Saunders, 2011.

4. Katzenstein AL. Katzenstein and Askin's surgical pathology of nonneoplastic lung disease. 4th ed. Philadelphia: Saunders Elsevier, 2006. 
5. Rosen Y. Four decades of necrotizing sarcoid granulomatosis: what do we know now? Arch Pathol Lab Med 2015; 139: 252-62.

6. Chittock DR, Joseph MG, Paterson NA, McFadden RG. Necrotizing sarcoid granulomatosis with pleural involvement: clinical and radiographic features. Chest 1994; 106: 672-6.

7. Sahin H, Ceylan N, Bayraktaroglu S, Tasbakan S, Veral A, Savas R. Necrotizing sarcoid granulomatosis mimicking lung malignancy: MDCT, PET-CT and pathologic findings. Iran J Radiol 2012; 9: 37-41.

8. Kim HJ. Current status of tuberculosis in Korea. Korean J Med 2012; 82: $257-62$

9. Joint Committee for the Development of Korean Guidelines for Tu- berculosis, Korea Centers for Disease Control and Prevention. Korean guidelines for tuberculosis. Seoul: Korea Centers for Disease Control and Prevention, 2011; 220.

10. American Thoracic Society; CDC; Infectious Diseases Society of America. Treatment of tuberculosis. MMWR Recomm Rep 2003; 52: $1-77$.

11. Harada T, Amano T, Takahashi A, et al. Necrotizing sarcoid granulomatosis presenting with elevated serum soluble interleukin-2 receptor levels. Respiration 2002; 69: 468-70.

12. Kim GS, Lee SJ, Lee JC, et al. A case of necrotizing sarcoid granulomatosis. Korean J Med 1997; 53: 574-9. 